\title{
Гетероструктуры CdHgТе для нового поколения ИК фотоприемников, работающих при повышенных температурах
}

\author{
() В.С. Варавин, В.В. Васильев, А.А. Гузев, С.А. Дворецкий, А.П. Ковчавцев, Д.В. Марин, И.В. Сабинина, \\ Ю.Г. Сидоров, Г.Ю. Сидоров, А.В. Царенко, М.В. Якушев
}

Институт физики полупроводников им. А.В. Ржанова Сибирского отделения Российской академии наук, 630090 Новосибирск, Россия

E-mail: yakushev@isp.nsc.ru

(Получена 27 апреля 2016 г. Принята к печати 10 мая 2016 г.)

\begin{abstract}
Приведены результаты исследования параметров многослойных гетероструктур $\mathrm{Cd}_{x} \mathrm{Hg}_{1-x}$ Те для фотоприемников диапазона длин волн до пяти микрометров, выращенных методом молекулярно-лучевой эпитаксии на кремниевых подложках. Методом $C-V$-характеристик исследованы пассивирующие свойства тонких слоев $\mathrm{CdTe}$ на поверхности таких структур. Исследованы температурные зависимости времени жизни неосновных носителей заряда в фотопоглощающем слое после роста и после отжигов. Фотодиоды $p^{+}-n$-типа были изготовлены ионной имплантацией мышьяка в слои $n$-типа проводимости, легированные In c концентрацией $(1-5) \cdot 10^{15} \mathrm{~cm}^{-3}$. Измерены температурные зависимости обратных токов при нескольких напряжениях смещения, которые оказались почти на 2 порядка меньше, чем у $n^{+}-p$-диодов.
\end{abstract}

\section{1. Введение}

Одной из важных задач современной микрофотоэлектроники является увеличение рабочей температуры охлаждаемых фотодиодных инфракрасных (ИК) фотоприемников. Более высокая рабочая температура позволяет снижать размер, вес, энергопотребление фотоприемных устройств (ФПУ) на их основе и расширять диапазон их применения [1]. Лидирующее место в производстве фотоприемников ближнего, среднего и дальнего ИК-диапазона занимают узкозонные полупроводниковые твердые растворы теллурида кадмия и ртути (КРТ). Особый интерес представляют гетероструктуры КРТ, выращенные на подложках из кремния, позволяющие снизить стоимость фоточувствительного материала и ФПУ на его основе. Такие гетероструктуры кардинально решают проблему согласования коэффициентов термического расширения кристалла с фотодиодами и кремниевой интегральной схемы считывания и предварительной обработки сигнала (мультиплексора), что, в свою очередь, упрощает производство ФПУ и существенным образом увеличивает их ресурс.

Для повышения рабочей температуры необходимо снизить величину темнового тока фотодиодов (ФД), определяющего их шум и обнаружительную способность. Обратный ток имеет несколько составляющих, связанных с различными физическими процессами в квазинейтральной и обедненной области ФД. Диффузионный ток зависит от температуры, как $\exp \left(-E_{g} / k T\right)$, генерационно-рекомбинационный ток пропорционален $\exp \left(-E_{g} / 2 k T\right)$. Токи утечек в местах выхода $p-n$-перехода на поверхность и некоторые другие составляющие тока имеют слабую температурную зависимость, но зависят от напряженности электрического поля в области пространственного заряда диода.

Цель данной работы заключается в исследовании свойств многослойных гетероструктур КРТ, выращен- ных на подложках $\mathrm{Si}(013)$ методом молекулярно-лучевой эпитаксии для ИК фотоприемников с длинноволновой границей спектральной чувствительности около 5 мкм, работающих при повышенных температурах.

\section{2. Результаты и обсуждение}

Рост гетероэпитаксиальных структур $\mathrm{CdHgTe} / \mathrm{Si}(310)$ осуществлялся в многокамерной сверхвысоковакуумной установке молекулярно-лучевой эпитаксии „Обь“ [2] с помощью разработанного технологического процесса, детали которого приведены в работе [3]. Для in situ контроля процессов предэпитаксиальной подготовки и роста использовались дифракция быстрых электронов (ДБЭ) и одноволновая эллипсометрия (длина волны $\lambda=632.8 \mathrm{Hм}$ ). В качестве подложек использовались кремниевые пластины КДБ 7-15 диаметром 76.2 мм, ориентированные по плоскости (310), производства $3 \mathrm{AO}$ „Телеком-СТВ“ (Зеленоград).

Схематическое изображение исследуемой структуры приведено на рис. 1. В качестве фотопоглощающего использован слой $n$-типа проводимости, полученный легированием индием, который не создает центров рекомбинации. Расположение $p$-области в широкозонной части гетероструктуры приводит к дополнительному заметному снижению темнового тока, что также позволяет повысить рабочую температуру ФД. Область $p$-типа проводимости может быть как однородным по толщине слоем постоянного состава, так и варизонным слоем с увеличивающимся к поверхности содержанием кадмия. Дополнительным достоинством таких структур является наличие на поверхности слоя CdTe, выполняющего функцию защитного диэлектрика. Этот слой выращивается в едином процессе создания всей гетероструктуры, что должно вести к низкой плотности поверхностных состояний на границе раздела $\mathrm{CdTe}-\mathrm{CdHgTe}$ и к уменьшению скорости поверхностной рекомбинации. Также 


\begin{tabular}{|c|}
\hline $\mathrm{CdTe}$ \\
thickness $0.25 \mu \mathrm{m}$; undoped \\
\hline$p-\mathrm{Cd}_{x} \mathrm{Hg}_{1-x} \mathrm{Te}$ \\
$0.35 \leq x \leq 0.6$; thickness $\leq 1 \mu \mathrm{m} ;$ \\
As doped at $1-10 \times 10^{17} \mathrm{~cm}^{-3}$ \\
\hline$n-\mathrm{Cd}_{x} \mathrm{Hg}_{1-{ }_{x} \mathrm{Te}}$ \\
$0.29 \leq x \leq 0.31 ;$ thickness $5 \mu \mathrm{m} ;$ \\
In doped at $1-10 \times 10^{15} \mathrm{~cm}^{-3}$ \\
\hline
\end{tabular}

Рис. 1. Схематическое изображение КРТ гетероструктуры.

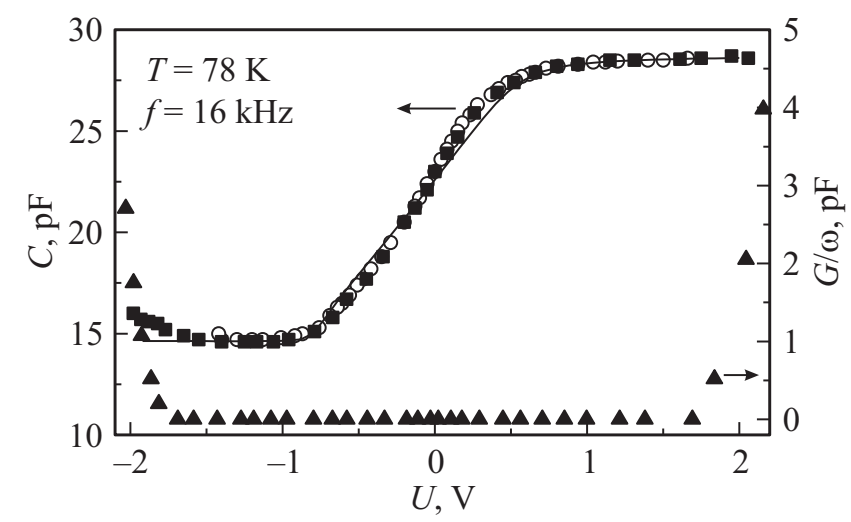

Рис. 2. Высокочастотная вольт-фарадная характеристика при прямом и обратном напряжении смещения $C-V$ и нормированная зависимость проводимости $G / \omega-V$ гетероструктуры $\mathrm{CdTe} / \mathrm{Cd}_{0.3} \mathrm{Hg}_{0.7} \mathrm{Te}$. Геометрические фигуры - эксперимент, сплошная кривая - расчет.

такие слои уменьшают токи утечки в местах выхода $p-n$-перехода на поверхность.

Для исследования пассивирующих свойств на слое $\mathrm{CdTe}$ формировались металлические электроды $(\mathrm{Ni} / \mathrm{In})$ площадью $A \approx 9 \cdot 10^{-4} \mathrm{~cm}^{2}$ и измерялись частотные зависимости адмиттанса. $C-V$-характеристики таких МДП-структур не имели заметной частотной зависимости в диапазоне $0.2-100$ кГц. При приложении к МДП-структурам пилообразного напряжения амплитудой от -3 до $3 \mathrm{~B}$ со скоростью изменения $0.5 \mathrm{~B} / \mathrm{Mин}$ на $C-V$-кривых отсутствовал гистерезис. На рис. 2 показана типичная экспериментальная $C-V$-кривая и нормированная кривая проводимости $G / \omega-V$. Сплошной линией показана расчетная $C-V$-зависимость, полученная с учетом изменения состава гетероструктуры по толщине пленки, и с учетом изменения ее зонной диаграммы с напряжением. Расчеты проводились в рамках диффузионно-дрейфовой модели. Из емкости в области инвертирующих смещений была оценена концентрация донорной примеси $N_{D} \approx 1.7 \cdot 10^{15} \mathrm{~cm}^{-3}$. Из емкости в обогащении оценена величина диэлектрической проницаемости слоя $\mathrm{CdTe} \varepsilon \approx 9.1$. Обращает на себя внимание достаточно хорошее совпадение между экспериментальной и теоретической кривыми, которое не требует обычно используемого приема - параллельного сдвига теоретической кривой по оси напряжений до совпадения с экспериментальной. Это указывает на отсутствие в МДП-структуре встроенных зарядов в слое CdTe и на отсутствие на границах раздела поверхностных состояний. Кривые нормированной проводимости на переменном сигнале $G / \omega$ в области напряжений смещения больше +1.6 и меньше $-1.6 \mathrm{~B}$ имеют резкий подъем, связанный, возможно, с утечками через пассивирующий слой CdTe.

Для выявления механизмов рекомбинации носителей заряда в пленках КРТ с $x \approx 0.3$ были исследованы температурные зависимости времени жизни после роста и после дополнительных отжигов. Отжиг для получения $p$-типа проводимости проводился при низком давлении паров ртути $\left(10^{-5}\right.$ атм $)$ в атмосфере инертного газа в течение $15-20$ ч при $T=250-260^{\circ} \mathrm{C}$. Двухстадийный отжиг в режиме активации мышьяка проводился при насыщенном давлении паров ртути (1-я стадия - 2 ч при $T=360^{\circ} \mathrm{C}$, 2-я стадия -24 ч при $\left.T=225^{\circ} \mathrm{C}\right)$. Первая стадия нужна для перевода атомов мышьяка в подрешетку теллура, в которой они становятся акцепторами, а вторая стадия необходима для заполнения вакансий ртути, образующихся на первой стадии.

В образцах после роста при низких температурах преобладает вклад рекомбинации Шокли-Рида (кривая 1 на рис. 3) с концентрацией глубоких уровней $N_{r} \approx 1 \cdot 10^{14} \mathrm{~cm}^{-3}$. После отжига для получения $p$-типа проводимости (за счет образования вакансий ртути) время жизни уменьшается по сравнению с образцом после роста и при температуре жидкого азота составляет

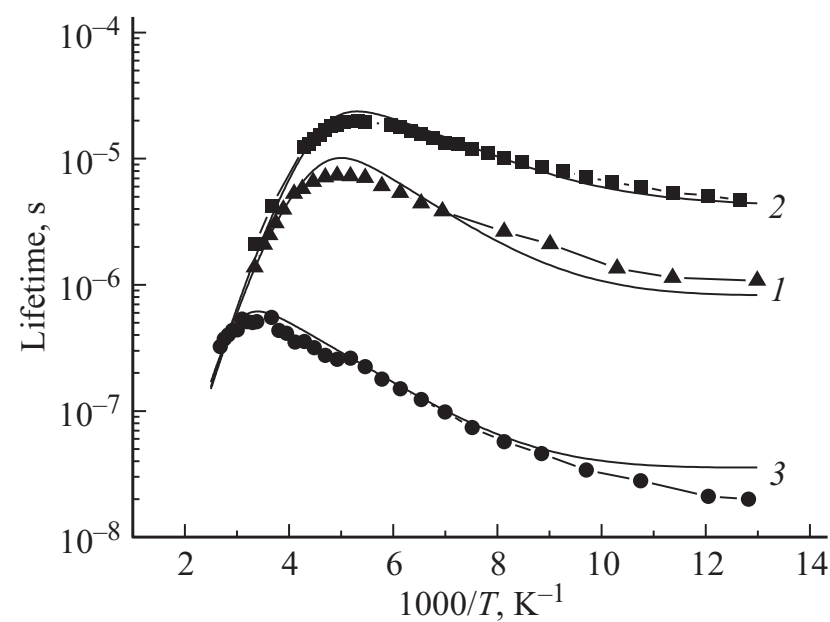

Рис. 3. Температурные зависимости времени жизни в гетероструктурах $\mathrm{Cd}_{0.3} \mathrm{Hg}_{0.7} \mathrm{Te} / \mathrm{Si}(013)$. Треугольники - экспериментальные значения в образце после роста, квадраты - после активационного отжига, круги - после отжига для получения $p$-типа проводимости. Сплошные кривые $1-3-$ расчет результирующего времени жизни с учетом рекомбинации излучательной, Оже и Шокли-Рида. 


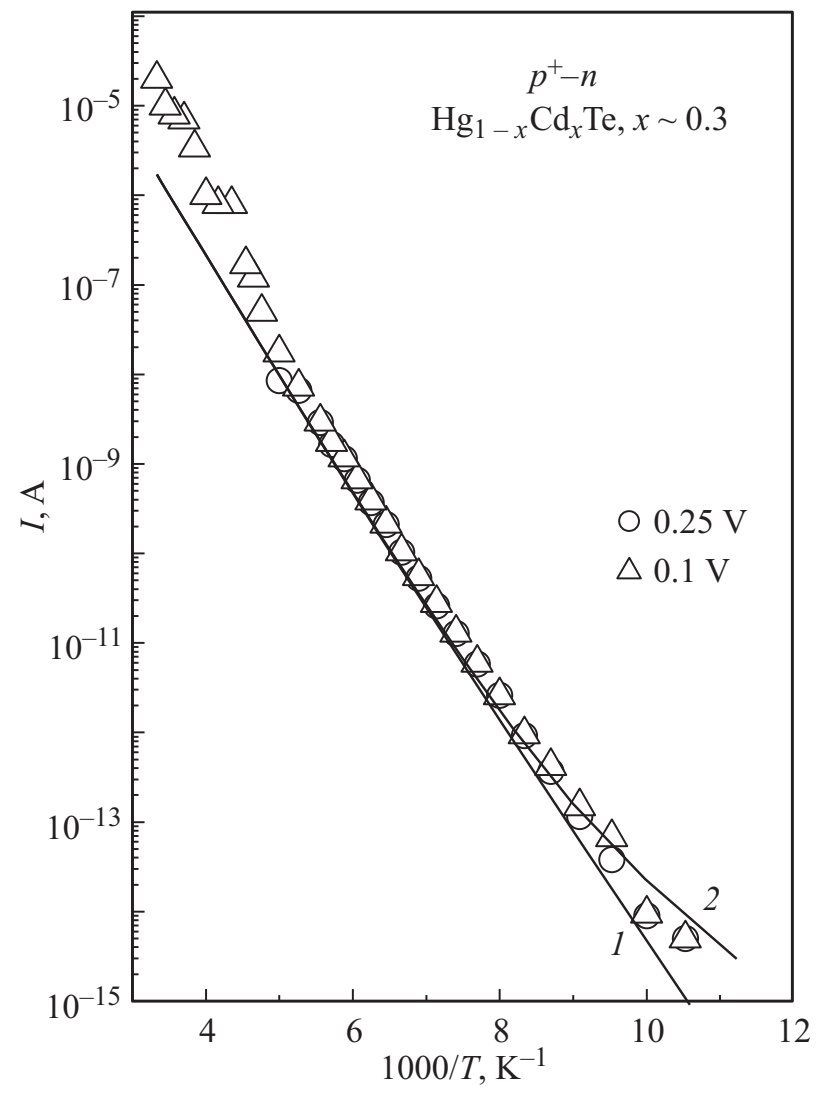

Рис. 4. Температурная зависимость обратного тока $p^{+}-n$ перехода площадью $1 \cdot 10^{-6} \mathrm{~cm}^{2}$ на гетероструктуре $\mathrm{Cd}_{0.3} \mathrm{Hg}_{0.7} \mathrm{Te} / \mathrm{Si}(013)$ в отсутствие освещения. 1 - расчетная зависимость диффузионной составляющей тока, 2 - расчетная зависимость с учетом диффузионной и генерационной составляющих тока. Геометрические фигуры - экспериментальные значения тока при двух напряжениях смещения на диоде.

несколько десятков наносекунд (кривая 3 на рис. 3). Температурная зависимость для этого случая описывается моделью с привлечением большой $\left(N_{r} \approx 1 \cdot 10^{15} \mathrm{~cm}^{-3}\right)$ концентрации глубоких уровней, связанных с вакансиями ртути. После активационного отжига, наоборот, время жизни при азотной температуре заметно возрастает (кривая 2 на рис. 3). Теоретически эта зависимость и зависимость после роста хорошо описываются моделью с привлечением глубоких уровней с одинаковой энергией, но с существенно меньшей (на порядок) концентрацией.

Для расчетов положение глубоких уровней бралось $E_{r}=0.055$ эВ выше потолка валентной зоны; при расчете оже-рекомбинации [4] интеграл перекрытия брался равным $\left|F_{1} F_{2}\right|=0.22$ и коэффициент $\gamma=3$; значения сечений захвата для электронов и дырок при расчете рекомбинации Шокли-Рида выбирались в диапазоне $\sigma_{n}\left(\sigma_{p}\right)=10^{-16}-10^{-15} \mathrm{~cm}^{2}$, что близко к данным работ $[5,6]$.

На основе легированных In гетероэпитаксиальных структур $\mathrm{Cd}_{x} \mathrm{Hg}_{1-x} \mathrm{Te}(x \approx 0.3)$ ионной имплантацией мышьяка с последующей активацией атомов мышьяка температурным отжигом были изготовлены матрицы планарных ФД $p^{+}-n$-типа и измерены температурные зависимости темнового тока отдельных ФД. На рис. 4 показаны экспериментальные и расчетные зависимости обратного тока от температуры в координатах Арpeниуса для двух значений напряжения на ФД (геометрические фигуры). Расчеты температурных зависимостей токов были выполнены на основе одномерной диффузионно-дрейфовой модели [7] с учетом ограничения токов диффузией подвижных носителей (кривая 1) и с учетом генерации носителей тока по механизму Са-Шокли-Рида-Холла (кривая 2). В области температур $T=120-210 \mathrm{~K}$ обратные токи ФД ограничивались диффузией неосновных носителей тока, а в области температур $T=95-120 \mathrm{~K}$ наблюдался избыточный ток. Избыточный ток при низких температурах можно объяснить вкладом термической генерации носителей через глубокий энергетический уровень, локализованный вблизи середины запрещенной зоны фоточувствительного слоя. Теоретическая кривая 2 была получена при концентрации глубоких уровней, равной $8 \cdot 10^{12} \mathrm{~cm}^{-3}$, и сечении захвата $\sigma_{n}=\sigma_{p}=1 \cdot 10^{-15} \mathrm{~cm}^{2}$. При более низких температурах ток становился меньше точности измерений.

Для сравнения ионной имплантацией бора в слои $p$-типа проводимости аналогичного состава были изготовлены матрицы ФД $n^{+}-p$-типа. Слои $p$-типа проводимости формировали введением в подрешетку металла необходимого числа вакансий с помощью температурного отжига в инертной атмосфере. Площадь ФД, как и в случае $n^{+}-p$-перехода, составляла $1 \cdot 10^{-6} \mathrm{~cm}^{2}$, шаг в матрице равнялся 30 мкм.

На рис. 5 показаны зависимости, аналогичные зависимостям на рис. 4, для $n^{+}-p$-ФД на основе вакансионного слоя $\mathrm{Cd}_{x} \mathrm{Hg}_{1-x}$ Te $(x \approx 0.3)$. В области температур $T=150-300 \mathrm{~K}$ обратные токи ФД ограничиваются диффузией неосновных носителей тока, а в области температур $T=77-150 \mathrm{~K}$ наблюдался избыточный ток, который при напряжениях обратного смещения $0.1-0.5 \mathrm{~B}$ достаточно хорошо объяснялся механизмом тепловой генерации неосновных носителей через глубокий энергетический уровень, локализованный вблизи середины запрещенной зоны фоточувствительного слоя. На рис. 5 кривыми 2 и 3 показаны теоретические зависимости обратных токов для двух напряжений смещения на ФД. При напряжениях смещениях $U>0.7 \mathrm{~B}$ зависимости экспериментальных токов от температуры становятся слабыми. Это может быть связано с проявлением механизма протекания туннельного тока утечки в местах выхода $n-p$-перехода на поверхность.

Из сравнения экспериментальных и расчетных температурных зависимостей токов была определена концентрация глубоких уровней, равная $1 \cdot 10^{15} \mathrm{~cm}^{-3}$, при сечении захвата $\sigma_{n}=\sigma_{p}=1 \cdot 10^{-15} \mathrm{~cm}^{2}$.

Следует отметить, что при одинаковом напряжении смещения величины токов $p^{+}-n$-диодов были примерно 


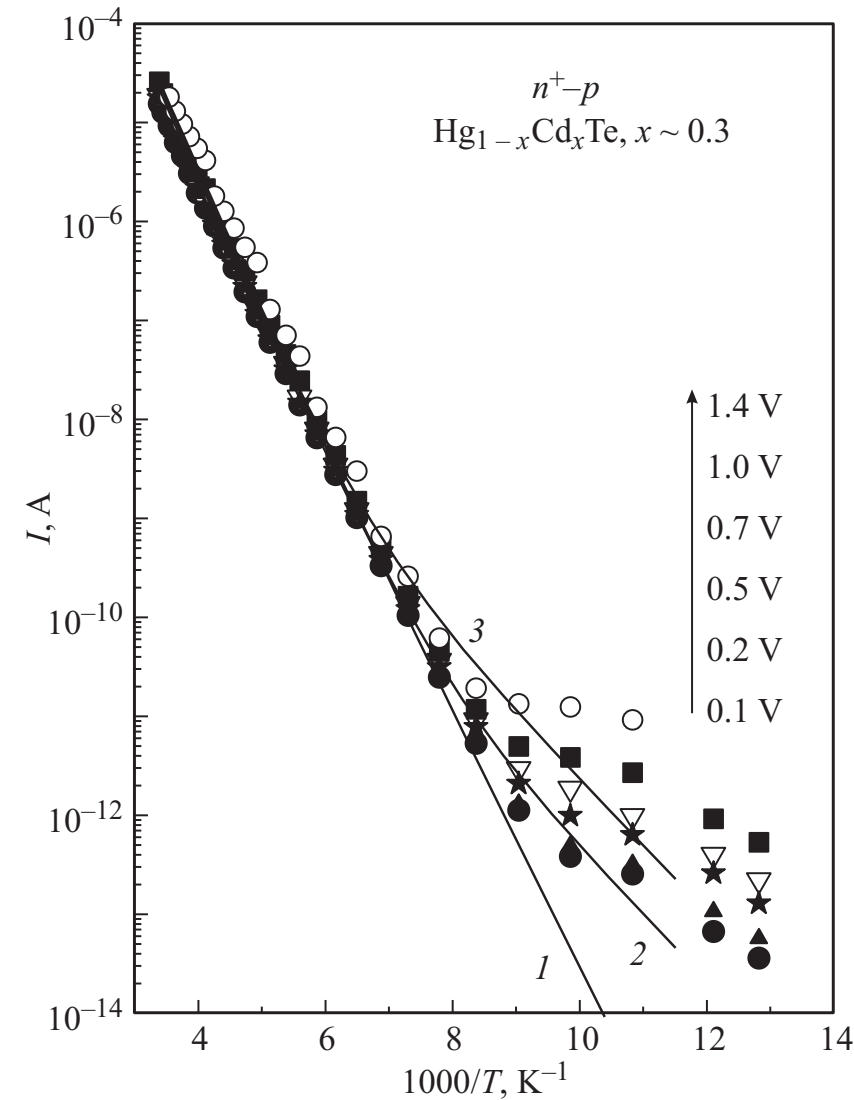

Рис. 5. Температурная зависимость обратного тока $n^{+}-p$ перехода на гетероструктуре $\mathrm{Cd}_{0.3} \mathrm{Hg}_{0.7} \mathrm{Te} / \mathrm{Si}(013)$ в отсутствие освещения. 1 - расчетная зависимость диффузионной составляющей тока, 2,3 - расчетная зависимость с учетом диффузионной и генерационной составляющей тока при двух обратных напряжениях на диоде $(0.1$ и $1.4 \mathrm{~B})$ соответственно. Геометрические фигуры - экспериментальные значения тока при нескольких напряжениях смещения на диоде.

на 2 порядка меньше, чем $n^{+}-p$-диодов на основе вакансионного слоя КРТ. Также можно отметить, что определение концентрации глубоких уровней из сопоставления экспериментальных и теоретических температурных зависимостей обратного тока несколько условно. Точно можно определить только произведение концентрации глубоких уровней на сечение захвата. Сечение захвата взято оценочно из литературных данных. Более строго концентрацию глубоких уровней можно определить из анализа величины емкости обратно смещенного диода большой площади. В нашем случае площадь диода составляла величину $1 \cdot 10^{-6} \mathrm{~cm}^{2}$, что приводило к малой величине емкости обратно смещенного диода и затрудняло проведение измерений.

\section{3. Заключение}

Защита поверхности фоточувствительного слоя $\mathrm{Cd}_{x} \mathrm{Hg}_{1-x} \mathrm{Te}(x \approx 0.3)$ варизонным слоем со слоем $\mathrm{CdTe}$ на поверхности снижает поверхностные утечки и темп поверхностной рекомбинации в фотодиодах и МДПструктурах. По результатам исследования $C-V$-характеристик МДП-структур с использованием широкозонного полупроводника CdTe в качестве диэлектрика можно заключить, что последний является хорошим защитным слоем, в котором отсутствует заметный встроенный заряд и на границе раздела $\mathrm{Cd}_{x} \mathrm{Hg}_{1-x} \mathrm{Te} / \mathrm{CdTe}$ нет заметной плотности поверхностных состояний.

Величины темновых токов в обратно смещенных $p^{+}-n$-фотодиодах оказались примерно на 2 порядка меньше, чем в $n^{+}-p$-фотодиодах. Это связано с низкой подвижностью дырок и низкой концентрацией глубоких рекомбинационных уровней в фоточувствительном $n$-слое КРТ, легированном индием. Следовательно, можно ожидать, что при одинаковых по сравнению с $n^{+}-p$-структурами значениями обнаружительной способности, ограниченной флуктуациями фонового потока, их рабочая температура может быть существенно выше.

Полученные результаты показывают, что, несмотря на большое рассогласование параметров кристаллической решетки КРТ и кремния, гетероструктуры $\mathrm{CdHgTe} / \mathrm{Si}(013)$, легированные индием, имеют низкую концентрацию рекомбинационных центров и могут быть использованы для разработки ИК детекторов, работающих при повышенных температурах.

Исследования выполнены при поддержке соглашения с Министерством образования и науки РФ RFMEFI60414X0134.

\section{Список литературы}

[1] A. Manissadjian, L. Rubaldo, Y. Rebeil, A. Kerlain, D. Brellier, L. Mollacd. Proc. SPIE, 8353, 835334-1 (2012).

[2] Ю.Г. Сидоров, С.А. Дворецкий, Н.Н. Михайлов, М.В. Якушев, В.С. Варавин, А.П. Анциферов. Оптич. журн., 67, 39 (2000).

[3] М.В. Якушев, Д.В. Брунев, В.С. Варавин, В.В. Васильев, С.А. Дворецкий, И.В. Марчишин, А.В. Предеин, И.В. Сабинина, Ю.Г. Сидоров, А.В. Сорочкин. ФТП, 45 (3), 396 (2011).

[4] V.C. Lopes, A.J. Syllaios, M.C. Chen. Semicond. Sci. Technol., 8, 824 (1993).

[5] P. Martyniuk, W. Gawron. Metrol. Meas. Syst., XXI (4), 675 (2014).

[6] P. Martyniuk, A. Kozniewski, A. Keblowski, W. Gawron, A. Rogalski. Opto-Electron. Rev., 22 (2), 118 (2014).

[7] A.P. Kovchavtsev, A.A. Guzev, A.V. Tsarenko, Z.V. Panova, M.V. Yakushev, D.V. Marin, V.S. Varavin, V.V. Vasilyev, S.A. Dvoretsky, I.V. Sabinina, Yu.G. Sidorov. Inf. Phys. Technol., 73, 312 (2015).

Редактор А.Н. Смирнов 


\title{
CdHgTe heterostructures for a new generation of infrared photodetectors operating at elevated temperatures
}

V.S. Varavin, V.V. Vasilyev, A.A. Guzev,

S.A. Dvoretsky, A.P. Kovchavtsev, D.V. Marin,

I.V. Sabinina, G.Yu. Sidorov, Yu.G. Sidorov,

A.V. Tsarenko, M.V. Yakushev

Rzhanov Institute of Semiconductor Physics

of Siberian Branch of Russian Academy of Sciences, 630090 Novosibirsk, Russia

\begin{abstract}
The results of studies of HgCdTe (MCT) multilayer heterostructures parameters for photodetectors of wavelengths range up to five microns grown on silicon substrates by molecular beam epitaxy are done. The properties of thin CdTe passivation layers, which were grown in single growth process on the surface are investigated by the $C-V$-measurements. The temperature dependences of the minority carrier lifetime in absorber layer of MCT heterostructures after the growth and the thermal annealing were investigated. The $p$-on- $n$ junctions were formed by As ions implantation into $n$-type absorber layer doped by In up to $(1-5) \cdot 10^{15} \mathrm{~cm}^{-3}$. We investigated the temperature dependences of diodes reverse current at several bias voltages. The reverse current for studied $p$-on- $n$ junction is two orders lower than for $n$-on- $p$ junction formed by B ions implantation in $p$-type vacancy doped MCT layers.
\end{abstract}

